
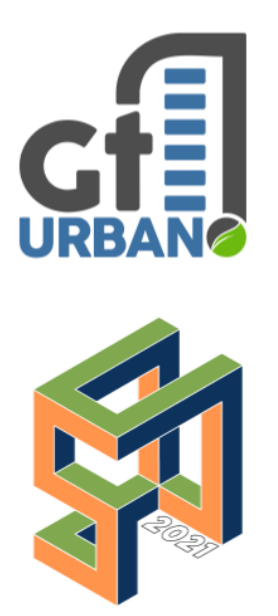

SINGEURB

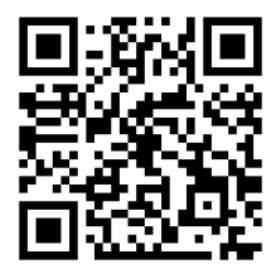

GONÇALVES, Luan

S. M; TEIXEIRA,

Bernardo A. N.

Identificação de

publicações sobre

dispositivos de

drenagem urbana

sustentáveis e chuvas

de projeto. In: III

SIMPÓSIO

NACIONAL DE

GESTÃO E

ENGENHARIA

URBANA:

SINGEURB, 2021,

Maceió. Anais...

Porto Alegre:

ANTAC, 2021. p. 79-

86.

Disponível em:

https://eventos.antac.

org.br/index.php/sin

geurb/issue/view/14

\title{
Identificação de publicações sobre dispositivos de drenagem urbana sustentáveis e chuvas de projeto
} Identification of publications on sustainable urban drainage techniques and design storms

Luan S. M Gonçalves, Universidade Federal de São Carlos, uansmg@hotmail.com

Bernardo A. N Teixeira, Universidade Federal de São Carlos, bernardo@ufscar.br

\section{RESUMO}

A recente urbanização brasileira seguiu um modelo de limitado planejamento urbano com grande densidade habitacional, pressionando a infraestrutura urbana. Desde a década de 80 , a gestão das águas urbanas tem evoluído do sistema tradicional higienista para abordagens mais sustentáveis, cumprindo imperativos hidrológicos, ecológicos, sociais e econômicos. Este trabalho identificou qual metodologia é mais frequente na determinação de chuvas de projeto e quais dispositivos de controle da água da chuva são mais estudados na área de drenagem sustentável, através de busca na base de dados Scopus. De um total de 7216 documentos avaliados, os poços de infiltração só foram mencionados em 3 trabalhos, e as bacias de detenção e infiltração foram citadas em 53 e 26 artigos, respectivamente. Já quanto as chuvas de projeto, os trabalhos científicos raramente fazem menção ao método utilizado, havendo um total de apenas 264 resultados positivos para todos os termos pesquisados, o que denota atribuição de baixa relevância para este importante parâmetro de dimensionamento e simulação. $\mathrm{O}$ baixo índice de pesquisas de dispositivos de drenagem comumente utilizados no Brasil indica a necessidade de intensificação dos estudos nacionais nesta área para melhoria da gestão dos riscos ambientais urbanos.

Palavras-chave: Gestão urbana, Hietogramas, Técnicas compensatórias.

\begin{abstract}
The recent Brazilian urban development followed a model of limited urban planning with high housing density, putting pressure on the city infrastructure. Since the 1980s, urban water management has evolved towards more sustainable approaches, fulfilling hydrological, ecological, social and economic imperatives. This work evaluated which methodology is often used in the determination of design storms and which technique for stormwater control are most studied in the area of sustainable drainage through a search in the Scopus database. From a total of 7216 documents analyzed, infiltration wells were only mentioned in 3 studies, and the detention and infiltration basins were mentioned in 53 and 26 articles, respectively. As for the design storms, the scientific works rarely mention the method used, with a total of only 264 positive results for all researched terms, which denotes a low relevance associated with this important parameter of design and simulation. The low rate of research on common
\end{abstract}


drainage devices used in Brazil indicates the need to intensify national studies in this area to improve the management of urban environmental risks.Qualquer dúvida envie um e-mail para "e-mail". (Nesta parte, os autores devem apresentar o resumo do artigo, em português, com no máximo 200 palavras).

Keywords: Urban management, Hyetograms, Alternative techniques.

\section{INTRODUÇÃO}

Quando ocorrem enchentes ou inundações, a população deseja que medidas rápidas sejam tomadas. O poder público precisa decidir onde e quanto investir para atender a comunidade, o que é reconhecidamente complicado devido a várias incertezas existentes. Um investimento vultoso para contenção de um problema de drenagem urbana pode ser desnecessário ou ineficaz se não houver um planejamento adequado.

Até meados do século 18, era aceitável o risco de inundações urbanas, sendo considerado um preço a se pagar pelo desenvolvimento próximo à água (BRASIL, 2007). No século 19, com o aumento da população citadina, tornou-se regra a canalização das águas urbanas de chuva, principalmente devido a relação direta entre saúde pública e construção de sistemas de drenagem (ASHLEY et al., 2015).

O sistema clássico de drenagem urbana, seguia um conceito higienista, e era projetado de forma a permitir a rápida evacuação das águas urbanas. No Brasil, o conceito higienista de "tudo para o esgoto" foi adotado efetivamente no final do século 19. Essa linha de pensamento prevê a canalização das águas pluviais, conduzindo a mesma preferencialmente no subterrâneo e por gravidade, permitindo o desenvolvimento urbano e a fácil circulação viária, evitando a presença da água na superfície e as possíveis doenças de veiculação hídrica (BRASIL, 2007).

O conceito higienista permaneceu em evidência até a década de 1970, quando o aumento do desenvolvimento urbano mostrou que essa concepção era insustentável e não era capaz de conter as enchentes. Foi necessário rever o sistema tradicional que possuía uma infraestrutura sobrecarregada, sendo necessário atuar na fonte ao invés de meramente conduzir a água, criando-se medidas de reservação e infiltração (MIGUEZ et al., 2015).

A drenagem urbana, historicamente uma disciplina da engenharia civil, desenvolveu-se então para abordagens integradas, ampliando as perspectivas para disciplinas e profissionais de arquitetura e urbanismo, gestão pública, ecologistas e sociólogos (FLETCHER et al., 2014).

Kim et al. (2017) observou que existem barreiras políticas, regulatórias, financeiras e de educação que dificultam a implementação de dispositivos de drenagem sustentáveis. Dhakal e Chevalier (2017) comentam que essas barreiras só serão superadas com o reconhecimento da comunidade que a drenagem sustentável é de fato benéfica para elas.

As técnicas compensatórias de drenagem urbana, tem como objetivo básico a retenção e infiltração das águas pluviais, melhorando a distribuição temporal das vazões escoadas em relação ao sistema clássico e, por consequência, provocando a diminuição ou inocorrência de inundações. Alguns destes dispositivos podem ser integrados ao meio urbano, permitindo diversos usos pela população (BAPTISTA et al., 2011). Andersen et al. (2017) observam que no dimensionamento de dispositivos de drenagem, os engenheiros utilizam uma chuva de projeto que representa o pior caso possível baseado em estatísticas pluviométricas. A chuva de projeto é a variável determinante nos volumes de detenção/retenção. 
Ao realizar um projeto de drenagem pode ser preciso assumir um hietograma de projeto. Hietogramas são uma representação gráfica da distribuição da chuva ao longo do tempo. Existe grande variabilidade nos tipos de hietogramas para precipitações de mesma duração. Balbastre-Soldevila et al. (2019) apresentam alguns hietogramas típicos utilizados em alguns países na Tabela 1. Os dois primeiros grupos são construídos diretamente a partir de relações intensidade-duração-frequência (IDF) das chuvas, enquanto o terceiro grupo é feito com perfis padrões de chuvas observados.

Tabela 1 - Modelos de Hietogramas

\begin{tabular}{ccc}
\hline Classificação & Modelo & País \\
& Hietograma & \\
\hline \multirow{2}{*}{ Padrão geométrico simples vinculado a um único ponto da } & Retangular & E.U.A. \\
curva IDF & Sifalda & Checoslováquia \\
& Triângulo Duplo & França \\
& Triangular & E.U.A. \\
& Linear/Exponencial & Canada \\
\hline Uso de toda a curva IDF & Blocos Alternados & E.U.A. \\
\hline Perfis padronizados de registros de chuva & ISWS & E.U.A. \\
& AVM & Australia \\
& NRCS & E.U.A. \\
& G2P & Espanha \\
\hline
\end{tabular}

Fonte: Adaptado de Balbastre-Soldevila et al. (2019)

A gestão da água pluvial em áreas urbanas necessita de soluções integradas tanto na superfície quanto no subsolo. As medidas na superfície exigem o uso inevitável de áreas com múltiplos interesses e atores envolvidos. Assim, a identificação dos aspectos relevantes para o ambiente urbano nos dá a oportunidade de desenvolver pesquisas que auxiliem a tomada de decisões.

Para verificar qual metodologia é mais frequente na determinação de chuvas de projeto e quais dispositivos são mais estudados na área de drenagem sustentável, foi realizada busca na base de dados Scopus, que é o maior banco de dados de resumos da literatura com revisão por pares, abrangendo revistas, livros, congressos entre outras publicações cientificas.

\section{MATERIAIS E MÉTODOS}

Na construção da busca na base Scopus, os termos de interesse foram pesquisados entre títulos, resumos e palavras-chave do banco de dados, especificados em língua inglesa e conectados com operadores booleanos "ou" para determinação de qualquer termo relacionado a drenagem sustentável, e o operador " $\mathrm{e}$ " para restringir o termo relacionado a chuva de projeto e a dispositivos de controle. A busca foi limitada a artigos, resumos e congressos. As Figuras 1 e 2 ilustram os termos utilizados na busca na base de dados para termos relacionados a chuva de projeto e dispositivos de controle de água pluvial sustentáveis. 
Figura 1 - Termos utilizados na busca por chuvas de projeto

\begin{tabular}{|c|c|c|c|c|c|}
\hline \multicolumn{6}{|c|}{ Termos relacionados a drenagem urbana sustentável } \\
\hline $\begin{array}{l}\text { Low Impact } \\
\text { Development }\end{array}$ & $\begin{array}{l}\text { ature-based } \\
\text { solutions }\end{array}$ & $\begin{array}{r}\text { Natu } \\
\text { sol }\end{array}$ & $\begin{array}{l}\text { Ised } \\
\text { Is }\end{array}$ & $\begin{array}{l}\text { Sustaina } \\
\text { Drainage }\end{array}$ & $\begin{array}{l}\text { le Urban } \\
\text { Systems }\end{array}$ \\
\hline $\begin{array}{c}\text { Green } \\
\text { infrastructure }\end{array}$ & \multicolumn{2}{|c|}{$\begin{array}{l}\text { Green and grey } \\
\text { infrastructure }\end{array}$} & \multicolumn{3}{|c|}{$\begin{array}{l}\text { Ecosystem-based disaster } \\
\text { risk reduction }\end{array}$} \\
\hline $\begin{array}{l}\text { Water Sensitive } \\
\text { cities }\end{array}$ & \multicolumn{2}{|c|}{$\begin{array}{l}\text { Integrated Urban } \\
\text { Water Management }\end{array}$} & \multicolumn{2}{|c|}{$\begin{array}{l}\text { Alternative } \\
\text { Techniques* }\end{array}$} & $\begin{array}{l}\text { Alternative } \\
\text { Techniques }\end{array}$ \\
\hline $\begin{array}{c}\text { Stormwater } \\
\text { Control Measures }\end{array}$ & \multicolumn{2}{|c|}{$\begin{array}{l}\text { Best Management } \\
\text { Practices* }\end{array}$} & \multicolumn{2}{|c|}{$\begin{array}{c}\text { Water Sensitive } \\
\text { Urban Design }\end{array}$} & $\begin{array}{l}\text { Sponge } \\
\text { Cities }\end{array}$ \\
\hline $\begin{array}{l}\text { Ecosystem-based } \\
\text { Adaptation }\end{array}$ & \multicolumn{2}{|c|}{$\begin{array}{l}\text { Integrated urban } \\
\text { water management }\end{array}$} & \multicolumn{2}{|c|}{$\begin{array}{c}\text { Green Blue } \\
\text { infrastructure }\end{array}$} & $\begin{array}{l}\text { Sponge } \\
\text { City }\end{array}$ \\
\hline $\begin{array}{l}\text { Total Encontrado: } \\
26639 \text { documentos }\end{array}$ & \multicolumn{5}{|c|}{$\begin{array}{l}\text { Total Encontrado sem os termos "Best } \\
\text { Management Pratice" e "Alternative } \\
\text { Techniques": } 7216 \text { documentos }\end{array}$} \\
\hline
\end{tabular}

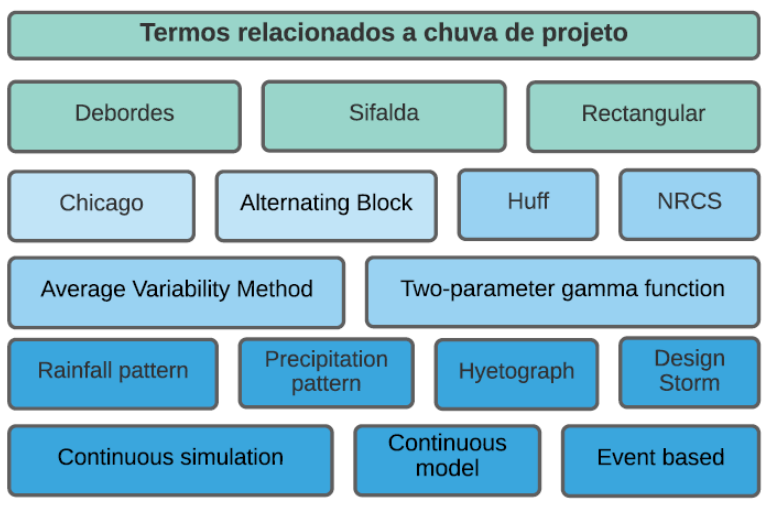

Fonte: Os autores

Figura 2 - Termos utilizados na busca por dispositivos de controle sustentáveis

\begin{tabular}{|c|c|c|c|c|c|}
\hline \multicolumn{6}{|c|}{ Termos relacionados a drenagem urbana sustentável } \\
\hline $\begin{array}{l}\text { Low Impact } \\
\text { Development }\end{array}$ & $\begin{array}{l}\text { Nature-based } \\
\text { solutions }\end{array}$ & $\begin{array}{l}\text { Nature } \\
\text { solu }\end{array}$ & $\begin{array}{l}\text { based } \\
\text { ons }\end{array}$ & $\begin{array}{l}\text { Sustainat } \\
\text { Drainage }\end{array}$ & $\begin{array}{l}\text { able Urban } \\
\text { e Systems }\end{array}$ \\
\hline $\begin{array}{c}\text { Green } \\
\text { infrastructure }\end{array}$ & \multicolumn{2}{|c|}{$\begin{array}{l}\text { Green and grey } \\
\text { infrastructure }\end{array}$} & \multicolumn{3}{|c|}{$\begin{array}{l}\text { Ecosystem-based disaster } \\
\text { risk reduction }\end{array}$} \\
\hline $\begin{array}{c}\text { Water Sensitive } \\
\text { cities }\end{array}$ & \multicolumn{2}{|c|}{$\begin{array}{c}\text { Integrated Urban } \\
\text { Water Management }\end{array}$} & \multicolumn{2}{|c|}{$\begin{array}{l}\text { Alternative } \\
\text { Techniques* }\end{array}$} & $\begin{array}{l}\text { Alternative } \\
\text { Techniques }\end{array}$ \\
\hline $\begin{array}{c}\text { Stormwater } \\
\text { Control Measures }\end{array}$ & \multicolumn{2}{|c|}{$\begin{array}{c}\text { Best Management } \\
\text { Practices }^{*}\end{array}$} & \multicolumn{2}{|c|}{$\begin{array}{l}\text { Water Sensitive } \\
\text { Urban Design }\end{array}$} & $\begin{array}{l}\text { Sponge } \\
\text { Cities }\end{array}$ \\
\hline $\begin{array}{l}\text { Ecosystem-based } \\
\text { Adaptation }\end{array}$ & \multicolumn{2}{|c|}{$\begin{array}{l}\text { Integrated urban } \\
\text { water management }\end{array}$} & \multicolumn{2}{|c|}{$\begin{array}{c}\text { Green Blue } \\
\text { infrastructure }\end{array}$} & $\begin{array}{l}\text { Sponge } \\
\text { City }\end{array}$ \\
\hline
\end{tabular}

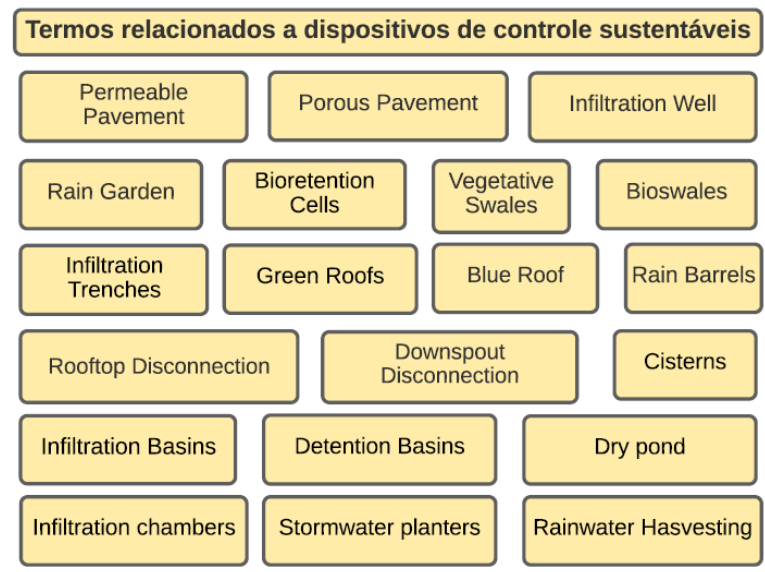

Fonte: Os autores

Um exemplo do protocolo de busca utilizado na plataforma: (TITLE-ABS-KEY("Low impact development") OR TITLE-ABS-KEY("Nature-based solutions") OR TITLE-ABS-KEY("Nature-Based Solutions") OR TITLEABS-KEY("Sustainable Urban Drainage Systems") OR TITLE-ABS-KEY("Water Sensitive Urban Design") OR TITLE-ABS-KEY("Green infrastructure") OR TITLE-ABS-KEY("Green blue infrastructure") OR TITLEABS-KEY("Ecosystem-based Adaptation") OR TITLE-ABS-KEY("Ecosystem-based disaster risk reduction") OR TITLE-ABS-KEY("Green and grey infrastructure") OR TITLE-ABS-KEY("integrated urban water management") OR TITLE-ABS-KEY("Stormwater Control Measures") OR TITLE-ABS-KEY("Water Sensitive cities") OR TITLE-ABS-KEY("Integrated Urban Water Management") OR TITLE-ABSKEY("Sponge Cities") OR TITLE-ABS-KEY("Sponge city") AND TITLE-ABS-KEY ( "Event Based" )) AND ( LIMIT-TO ( DOCTYPE,"ar" ) OR LIMIT-TO ( DOCTYPE,"cp" ) OR LIMIT-TO ( DOCTYPE,"re" ) ). 
Não foi realizada limitações quanto a língua e data de publicação, já que o objetivo é analisar a quantidade de produções cientificas que trazem em seu título, resumo e palavras-chave a definição do tipo de chuva de projeto que está sendo utilizado no estudo e não realizar estudo aprofundado de cada uma dessas publicações. Quanto a área científica de publicação não é possível realizar restrições, já que o caráter de multidisciplinaridade da drenagem urbana sustentável possui artigos classificados desde áreas médicas até a área da computação. As aspas são aplicadas aos termos para busca por palavras escritas exatamente daquela forma.

Dentre os termos relacionados a drenagem sustentável, os termos alternative techniques e best management practices foram excluídos por serem termos genéricos e vincular resultados pesquisas em todos os campos científicos e não apenas a drenagem urbana. Para os termos relacionados a chuva de projeto, buscou-se por palavras relacionadas aos hietogramas mais utilizados, e a modelagens contínuas e baseadas em eventos. A busca pelos termos de dispositivos de drenagem sustáveis mais utilizados usou a mesma estratégia de pesquisa adotada para as chuvas de projeto.

\section{RESULTADOS}

$\mathrm{Na}$ busca inicial considerando todos os termos propostos relacionados a drenagem urbana sustentável, inclusive com as palavras alternative techniques e best management practices foram encontrados 26639 documentos com menções aos termos. Após a exclusão do termo best management practices a busca retornou 17914 arquivos e após a exclusão do termo alternative techniques a busca se limitou a 7216 documentos. Figura 3 mostra os resultados obtidos para cada termo relacionado a chuva de projeto.

Figura 3 - Menções para os termos de chuva de projeto na base Scopus

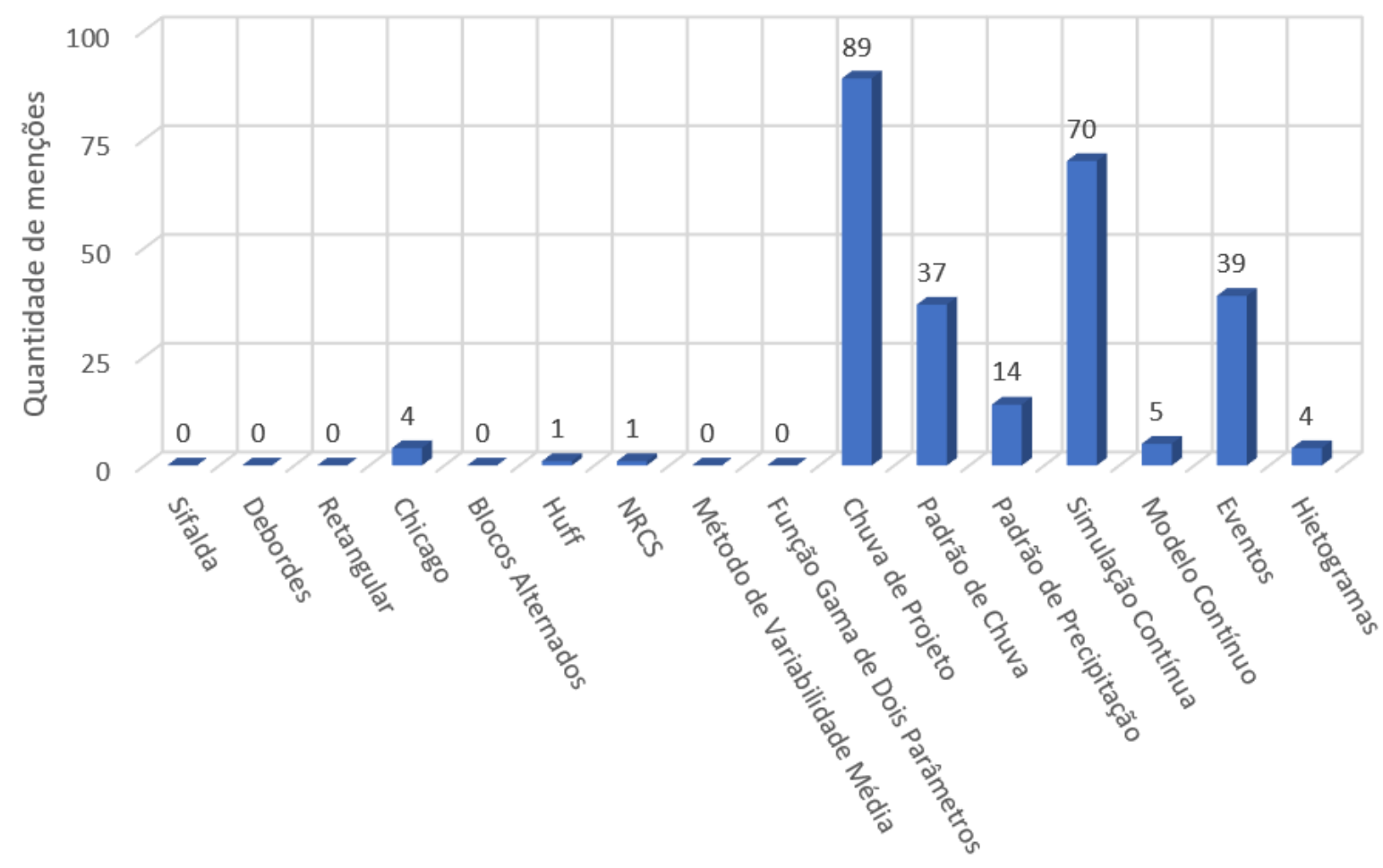

Fonte: Os autores 
Para os termos "Chicago", "NRCS", "Retangular" foi feita leitura dos resumos para confirmação da relação das palavras a chuvas de projeto, já que NRCS é uma agência americana, Chicago é uma cidade de grande porte americana e retangular é uma forma geométrica, o que poderia sugerir resultados não aderentes. Para "Chicago" das 35 menções encontradas apenas 4 se relacionavam a chuvas, já quanto o termo "NRCS" dos 36 encontrados apenas 1 fazia menção a chuva de projeto, e por fim o termo "retangular" não possuiu nenhum resultado aderente dos 6 encontrados.

De forma geral, a quantidade de documentos científicos relacionados a drenagem sustentável que trazem em seu título, resumo ou palavras-chave termos relacionados a chuvas de projeto é muito baixa. Os hietogramas de base geométrica (Debordes, Sifalda e Retangular) estão ausentes, os de base em toda curva IDF (Chicago e Blocos Alternados) aparecem apenas em 4 artigos e os hietogramas de perfis padronizados (Huff, NRCS, Método de Variabilidade Média e Função Gama de Dois Parâmetros) estão presentes em apenas 2 documentos. O próprio termo hietograma só aparece em 4 artigos.

Quanto aos termos chuva de projeto, padrão de chuva e padrão de precipitação, a apresentação é um pouco maior, mas ainda assim, caso fossem somadas estariam presentes em 140 publicações, representando apenas 1,94\% da amostra total. Já os termos de simulação contínua ou modelo contínuo representam 1,04\% do total e o termo de simulação baseada em eventos representa $0,54 \%$ dos documentos pesquisados.

Quanto aos dispositivos de controle de água de chuva sustentáveis, a Figura 4 mostra a quantidade de menções para cada termo.

Figura 4 - Menções para os termos de dispositivos sustentáveis na base Scopus

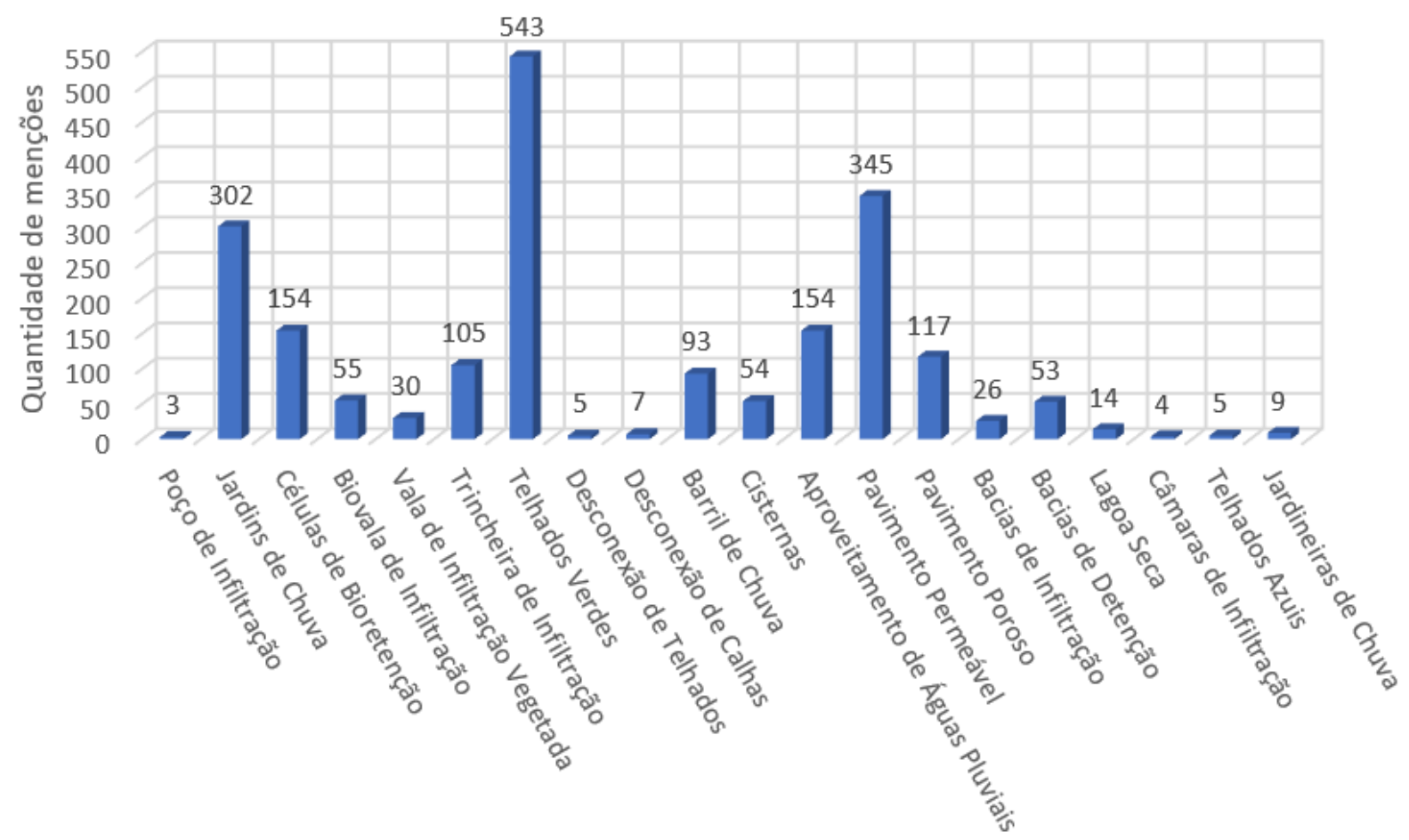

Fonte: Os autores 
Observa-se grande interesse nos termos relativos a pavimentos permeáveis, que juntos representam 462 menções. Os telhados verdes apresentam o maior interesse das publicações obtendo 543 menções. As trincheiras de infiltração e células de bioretenção/biovala de infiltração apresentam interesse intermediário, aparecendo em 105 e 209 publicações respectivamente.

As bacias de detenção e infiltração, muito utilizadas no Brasil, estão com baixa associação a termos sustentáveis, com 53 e 26 menções. Outro dispositivo de grande utilização no Brasil, os poços de infiltração, possui apenas 3 artigos no banco de dados Scopus para a amostra selecionada.

O termo aproveitamento de águas pluviais associado aos termos barril de chuva e cisternas representam somados 301 menções, mostrando grande interesse nas técnicas de aproveitamento. Outras técnicas como os telhados azuis, câmaras de infiltração, desconexão de telhados e vala de infiltração vegetada possuem baixo interesse dos pesquisadores.

\section{CONCLUSÕES}

A baixa indicação da chuva de projeto nos pontos de destaque das publicações pode estar relacionada a uma atribuição de baixa relevância da representação estatística da chuva e de como essa chuva influencia na estimativa do desempenho real dos dispositivos de drenagem sustentáveis.

Quanto aos dispositivos de drenagem sustentável, é surpreendente o baixo índice de publicações com poços de infiltração, uma das soluções mais comuns no Brasil para pequenos e médios lotes urbanos, o que mostra a necessidade de desenvolvimento de pesquisas nacionais que contemplem esta realidade brasileira. As trincheiras de infiltração, os microreservatórios e os planos de infiltração, que possuem relevância intermediária nas publicações e que são comuns de serem aplicados em cidades brasileiras, também necessitam de maior atenção do meio cientifico.

Este trabalho identificou algumas lacunas de conhecimento na área de drenagem urbana sustentável que geram impacto na gestão dos riscos hidrometeorológicos urbanos. O preenchimento destas lacunas ajudará as cidades a melhorar a capacidade dos seus sistemas de drenagem e a reduzir os riscos ambientais associados.

\section{REFERÊNCIAS}

ANDERSEN, J. S. et al. Characteristic Rain Events: A Methodology for Improving the Amenity Value of Stormwater Control Measures. Sustainability, v. 9, n. 1793, 2017.

ASHLEY, R. M. et al. UK Sustainable Drainage Systems: past, present and future. Proceedings of ICE - Civil Engineering, v. 168, p. 125-130, 2015.

BALBASTRE-SOLDEVILA, R.; GARCÍA-BARTUAL, R.; ANDRÉS-DOMÉNECH, I. A Comparison of Design Storms for Urban Drainage. Water, 2019.

BAPTISTA, M.; NASCIMENTO, N.; BARRAUD, S. Técnicas Compensatórias em Drenagem Urbana. 2ª ed. Porto Alegre: Associação Brasileira de Recursos Hídricos, 2011. 
BRASIL. Águas pluviais: técnicas compensatórias para o controle de cheias urbanas: guia do profissional em treinamento nível 2 e 3. Belo Horizonte: Ministério das Cidades , 2007. 52 p.

DHAKAL, K. P.; CHEVALIER, L. R. Managing urban stormwater for urban sustainability: Barriers and policy solutions for green infrastructure application. Journal of Environmental Management, 2017. 171-181.

FLETCHER, T. D. et al. SUDS, LID, BMPs, WSUD and more - The evolution and application of terminology surrounding urban drainage. Urban Water Journal, v. 12, 2014.

KIM, J.-H.; KIM, H. Y.; DEMARIE, F. Facilitators and Barriers of Applying Low Impact Development Practices in Urban Development. Water Resources Management, 2017. 3795-3808.

MIGUEZ, M. G.; VERÓL, A. P.; REZENDE, O. M. Drenagem Urbana: Do Projeto Tradicional à Sustentabilidade. 1ํ. ed. [S.1.]: Elsevier, 2015. 